\title{
Bekenntnisse eines SP
}

Bernhard Gurtner

Korrespondenz:

Dr. med. Bernhard Gurtner Eggstrasse 76 CH-8620 Wetzikon

gurtner.bernhard@bluewin.ch
Keine Sorge, es geht hier nicht um Politik. SP ist die gebräuchliche Abkürzung für Schauspielpatienten, die im Medizinstudium für die Schulung und Prüfung der kommunikativen Fähigkeiten eingesetzt werden. In den USA begann man damit schon vor 50 Jahren, in Europa mit bedächtiger Skepsis etliches später. Die didaktisch eingesetzten Simulanten haben sich als wertvolle Hilfe erwiesen, könnten nun aber vielerorts dem Spardruck und dem Unverständnis einiger Budgetverantwortlicher zum Opfer fallen, die ihre Kredite lieber für teure Geräte als für billige Komödianten reservieren möchten. Sie verkennen, wie kostendämpfend gute ärztliche Gespräche sind. Umfassende Anamnesen ersparen aufwendige diagnostische Irrwege. Zudem wird eine vertrauensvolle therapeutische Beziehung durch gepflegte Kommunikation nachhaltiger begründet als durch hochtrabende Werbeprospekte glanzvoller Kliniken.

Hier soll deshalb von den Erfahrungen eines ungenannt bleibenden SP berichtet werden, der seinen Teilzeit-Job vor einigen Jahren mit grosser Skepsis und einigen Hemmungen übernommen hat, ihn aber ungern wieder aufgeben würde. Er habe sich damals auf ein Inserat der Medizinischen Fakultät gemeldet, weil er als arbeitsloser Schauspieler seine Talente nicht nur für Werbespots, sondern auch für etwas Vernünftigeres einsetzen wollte. Ein ausreichender Zusatzverdienst sei zwar nicht zu gewinnen, jedoch eine Menge neuer Erlebnisse, nicht nur mit den angehenden Ärztinnen und Ärzten, sondern auch mit den aus verschiedenen Berufen stammenden LaienDarsteller(inne)n. Die jüngste sei 18-jährig und spiele gerne die neurovegetativ labile, verwöhnte Tochter; der älteste SP, Mitglied einer Seniorentheatergruppe, sei bereits 81 und sehr gefragt als authentischer Darsteller eines schwerhörigen Besserwissers, wie sie unter den echten Patienten dieser Altersgruppe ja nicht selten sind.
Die Instruktion und Supervision der SP erfolge durch erfahrene Kaderärzte und psychologisch geschulte Kommunikationstrainer, Stegreiftheater sei nicht erwünscht. Es werden Rollen mit einer glaubhaften Sozialanamnese und den typischen Beschwerden einer bestimmten Erkrankung eingeübt. In den Kursen selbst gehe es weniger darum, dass die Studierenden wie Detektive mit gezielten Fragen zu einer Diagnose gelangen, vielmehr sollen sie sich durch geduldiges Zuhören ein Bild von der Persönlichkeit und Lebensgeschichte des Patienten verschaffen. Wer-hat-was? sei wichtiger als Was-hat-eine(r)? Die Lernenden erproben auch, mit schwierigen Patienten zurechtzukommen. Den verstockten oder überaus aggressiven Patienten spiele unser Gewährsmann besonders gerne, einmal so echt, dass er bei einer empfindsamen Gesprächspartnerin Tränen provoziert habe.

Viel mehr Mühe mache es ihm jeweils, schlechte Nachrichten - Bad News sei die fachübliche Bezeichnung - mit echt wirkender Betroffenheit entgegenzunehmen. Die Medizinstudentin erkläre ihm zum Beispiel zögernd, dass er einen unheilbaren Krebs habe. Soll er nun schockiert, verzweifelt, ungläubig, wütend oder sprachlos reagieren? Das gelinge nicht immer, weil beide Beteiligten um die Irrealität der Situation wüssten. Das Rollenspiel komme der Wirklichkeit aber oft doch erstaunlich nahe und diene in der anschliessenden gemeinsamen Besprechung als Lehrstück für angemessenes ärztliches Verhalten. Videoaufzeichnungen verhelfen den Studierenden und den SP gleichermassen zur Selbstkritik der verbalen und nicht verbalen Kommunikation. Die Aufnahmen liefern ein jederzeit verwendbares Schulungsmaterial, ohne dass reale Patienten wiederholt belästigt werden müssen.

Geübt werde von den Studierenden auch die oft vernachlässigte Sexualanamnese, was der auf- und abgeklärten akademischen Jugend gar nicht so leicht

\section{Konsultation anstatt Komödie}

«I'm the gastric ulcer», flüsterte eine von Hollywood heruntergekommene Schauspielerin dem Medizinstudenten ins Ohr, nachdem sie bei der Anamnese dem Drehbuch getreu über Nüchternschmerzen und schwarzen Stuhl berichtet und bei der Palpation des Oberbauches mit einem schrecklichen Schrei reagiert hatte. Dann streifte die nun als echte Patientin agierende Frau ihre Strümpfe nach unten und fragte den jungen Mann, was sie gegen ihre üblen Varizen tun könnte. Sie spürte, dass sich ein guter künftiger Arzt mit ihr befasste, und schenkte ihm Vertrauen, obwohl sie wusste, dass er noch kein echter Doktor war.

1986 beobachtet in der Division of Research in Medical Education der University of Southern California in Los Angeles (Prof. Stephen Abrahamson). 


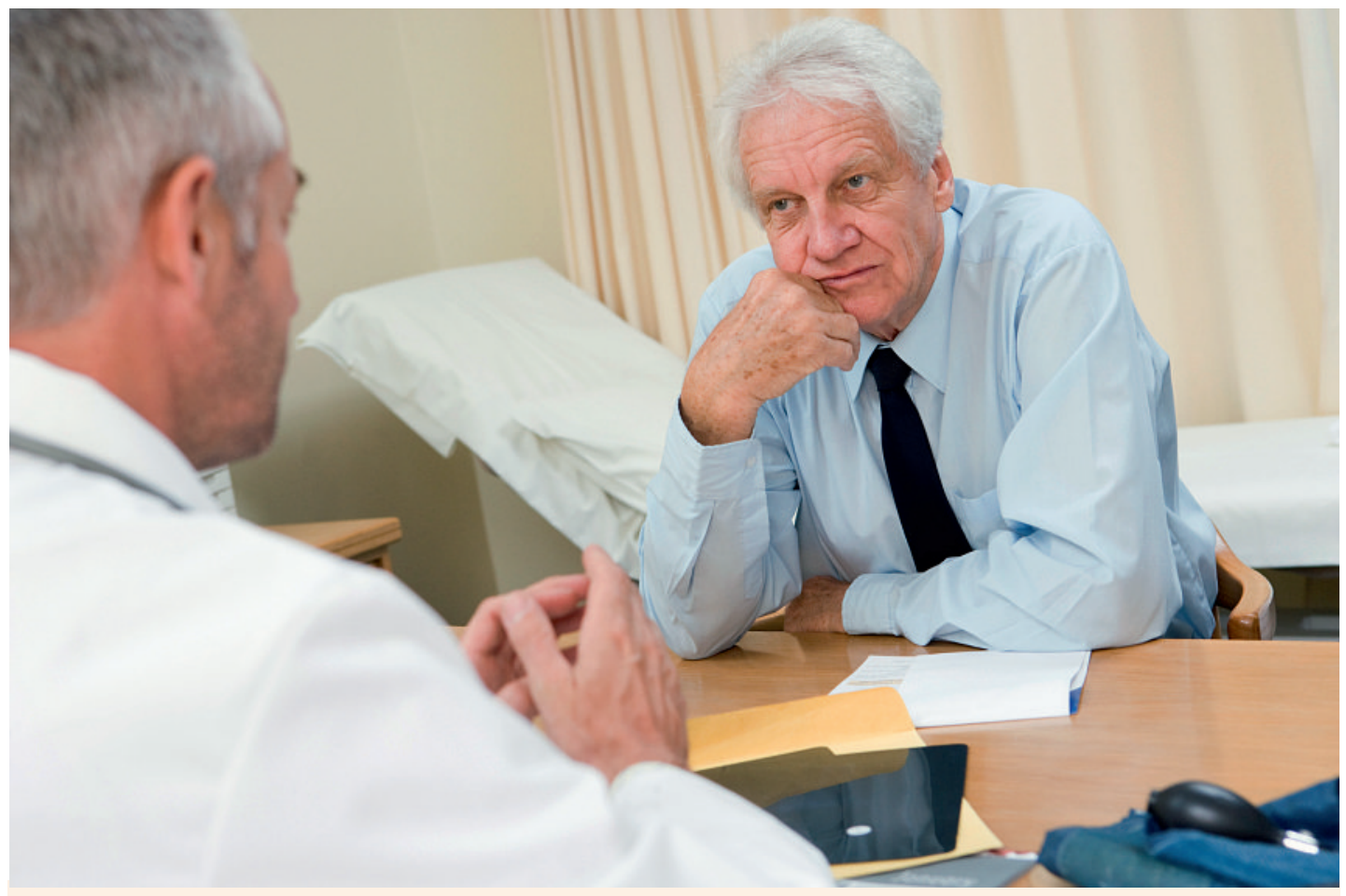

Eine besondere Herausforderung für den SP: das Entgegennehmen von «Bad news» mit echt wirkender Betroffenheit.

falle, wie sich unser SP als spätmittelalterlicher Mann gedacht hätte. Das hinlängliche Vokabular und die ersten persönlichen Erfahrungen seien zwar vorhanden, doch erfolgten die Fragen an die SP entweder so unverschämt forsch wie von einem Reporter der Regenbogenpresse oder doch irgendwie verklemmt wie anno dazumal. Ob der (angebliche) Krebspatient seelischen Beistand erhalte oder wünsche, werde in der
In Grossbritannien werden SP vom nationalen Gesundheitsdienst als getarnte Qualitätsprüfer in Spitäler oder Arztpraxen eingeschmuggelt, wo sie nur zu 10-20\% entlarvt werden. Bei uns gibt es (noch?) keinen medizinischen Gault-Millau, doch hat nun der SP seinen eigenen Arzt kritischer beobachtet. Er kennt ihn als sympathischen Mann, der ihm rasch und grosszügig Medikamente abgibt, jedoch kaum

\section{In Grossbritannien werden SP vom nationalen Gesundheitsdienst als getarnte Qualitätsprüfer in Spitäler oder Arztpraxen eingeschmuggelt, wo sie nur zu 10-20\% entlarvt werden.}

Spielsituation kaum je gefragt, die spirituelle Anamnese sei kein Thema.

Nun also werde der SP-Einsatz durch Sparmassmahmen bedroht. Als Ersatz könnten die Studierenden nach Meinung einiger Kliniker gratis Doktorspiele üben und sich abwechselnd als Arzt oder Patient darstellen. Das wäre immer noch besser als gar kein Training des Kommunikationsverhaltens. einmal danach fragt, welche familiären oder beruflichen Probleme das Herzflattern ausgelöst haben könnten. Bei jeder Konsultation sei er vordringlich damit beschäftigt, mit dem PC zurechtzukommen, und gönne ihm nur selten einen kurzen Augenblick. Bei der nächsten Konsultation würde er ihm dazu gerne ein professionelles Feed-back geben, doch dann laufe er wohl Gefahr, in den Akten inskünftig als SP (= schwieriger Patient) vermerkt zu bleiben. 